\title{
Matter Symmetries of Linet-Tian Metric in General Theory of Relativity
}

\author{
R. S. Rane', S. S. Dabhane ${ }^{2}$ \\ ${ }^{1}$ Department of Mathematics, Y. C. Arts and Science College, Mangrulpir \\ ${ }^{2}$ Department of Mathematics, Dr. Sau. Kamaltai Gawai Institute of Engg. \& Tech., Darapur
}

\begin{abstract}
In this paper, we have discussed the symmetries of the energy-momentum tensor $\left(T_{a b}\right)$ for the Linet-Tian metric. We have obtained different constraint equations on energy-momentum tensor $\left(T_{a b}\right)$. If we solve these constraint equations, we obtain new exact solutions of Einstein field equations. We have solved matter symmetries (collineations) equations for the four main cases by taking one, two, three and four non-zero components of the energy-momentum tensor $\left(T_{a b}\right)$. We have investigated the degenerate case i.e. $\operatorname{det}\left(T_{a b}\right)=0$ for one, two and three non-zero components energy-momentum tensor $\left(T_{a b}\right)$ and non-degenerate case i.e. $\operatorname{det}\left(T_{a b}\right) \neq 0$ for all four components of energy-momentum tensor $\left(T_{a b}\right)$ are non-zero. It is observed that, this space-time have infinite number of matter symmetries (collineations) in degenerate case and have finite number of matter symmetries in non-degenerate case. It is also observed that this space-time gives seven independent matter symmetries in which three are linearly independent Killing vectors and the remaining are dependent.
\end{abstract}

Keywords: Linet-Tian metric, matter symmetries (collineations), energy-momentum tensor

\section{Introduction}

In general theory of relativity, the gravitational field is described by the Einstein tensor which is contained in the curvature of space-time via Ricci tensor and Ricci scalar. As Ricci tensor enables us to understand the geometric structure of space-time, the energy-momentum tensor plays a significant role in understanding physical structure of spacetime. The gravitational field possesses the symmetries which are expressed in terms of Killing vector fields. The matter contained is represented by energy-momentum tensor. The space-time geometry is related to the matter contained through Einstein field equations [1]. The Einstein's field equations (EFEs) are given by

$$
G_{i j} \equiv R_{i j}-\frac{1}{2} R g_{i j}=\kappa T_{i j},
$$

where $G_{i j}$ are the components of the Einstein tensor, $R_{i j}$ are Ricci tensor and $T_{i j}$ of the matter (energy-momentum) tensor. Also, $R=g^{i j} R_{i j}$ is the Ricci scalar, and $\Lambda=0$ for simplicity. The symmetries (collinetaions) play an important role in Einstein's field equations (1) of general relativity [25]. These symmetries can be expressed $L_{X} \boldsymbol{A}=\boldsymbol{B}$ where $\boldsymbol{A}$ and $\boldsymbol{B}$ are the geometric /physical fields, $\boldsymbol{X}$ is a vector field generating the symmetry and $L_{X}$ denotes Lie derivative operator along with vector field $\boldsymbol{X}$.

A vector field $\mathbf{X}$ is an matter collineation for matter tensor $T_{i j}$ if

$$
\mathcal{L}_{X} T_{a b}=0 \Leftrightarrow T_{a b, c} X^{c}+T_{a c} X_{, b}^{c}+T_{b c} X_{, a}^{c}=0 .
$$

In last decades, some authors [6-13] shows keen interest in study of matter symmetries (collineations). M. Sharif [1415] has studied the cylindrically symmetric static space-time and static space-time with maximal symmetric transverse spaces according to their matter collineation. M. Salti et al [16] have investigated matter collineation of BKS-type space-time.

In this paper, we have studied the matter symmetry properties of Linet-Tian metric with different constraint conditions on $T_{i j}$. In section 2.1, we have classified LinetTian metric with degenerate case i.e. $\operatorname{det}\left(T_{a b}\right)=0$ by considering one, two and three non-zero components of $T_{a b}$. It is observed that, there are infinite numbers of matter symmetries (collineations) in degenerate case. In section 2.2, we have discussed the non-degenerate case i.e. $\operatorname{det}\left(T_{a b}\right) \neq 0$ by considering four non-zero components of $T_{a b}$. In this case, there are finite number of matter symmetries. It is also shown that this space-time gives seven independent matter symmetries in which three are linearly independent Killing vectors and the remaining are dependent. Later, in section 3, we have discussed the obtained results. Lastly, conclusion is given in section 4 .

\section{Matter Symmetries (collineations) Equations}

Consider Linet-Tian [17-18] space-time is expressed as

$$
d s^{2}=-f d t^{2}+d \varrho^{2}+g d z^{2}+l d \phi^{2},
$$

where

$$
\begin{gathered}
f=Q^{2 / 3} P^{-2\left(1-8 \sigma+4 \sigma^{2}\right) / 3 \Sigma}, \\
g=Q^{2 / 3} P^{-2\left(1+4 \sigma-8 \sigma^{2}\right) / 3 \Sigma}, \\
l=c^{2} Q^{2} P^{4\left(1-2 \sigma-2 \sigma^{2}\right) / 3 \Sigma} .
\end{gathered}
$$

where $t, \varrho, z, \phi$ are usual cylindrical co-ordinates, $\sum=1-$ $2 \sigma+4 \sigma^{2}$, the constant $\sigma$ is related but not equal to mass per unit length, the constant $c>0$ is related to angle defect and for $\wedge<0$,

$$
P=\frac{2}{\sqrt{3|\wedge|}} \tan h(R), Q=\frac{1}{\sqrt{3|\wedge|}} \sin h(2 R), R=\frac{\sqrt{3|\wedge|}}{2} \varrho
$$

In the limit $\wedge \rightarrow 0$, the metric reduces to the Levi-Civita metric for which $P=Q=\varrho$.

The non-zero components of Ricci-Tensor are

$$
\begin{aligned}
& R_{00}=-\frac{f^{\prime \prime}}{2}+\frac{f^{\prime 2}}{4 f}-\frac{f^{\prime} l^{\prime}}{4 l}-\frac{f^{\prime} g^{\prime}}{4 g}, \\
& R_{11}=-\frac{f^{\prime \prime}}{2 f}+\frac{g^{\prime \prime}}{2 g}+\frac{l^{\prime \prime}}{2 l}-\frac{f^{\prime 2}}{4 f^{2}}-\frac{g^{\prime 2}}{4 g^{2}}-\frac{l^{\prime 2}}{4 l^{2}}, \\
& R_{22}=\frac{g^{\prime \prime}}{2}-\frac{g^{\prime 2}}{4 g}+\frac{f^{\prime} g^{\prime}}{4 f}+\frac{g^{\prime} l^{\prime}}{4 l}, \\
& R_{33}=\frac{l^{\prime \prime}}{2}+\frac{f^{\prime} l^{\prime}}{4 l}+\frac{f^{\prime} g^{\prime}}{4 g},
\end{aligned}
$$

where prime denotes differentiation with respect to $\varrho$. 


\section{International Journal of Science and Research (IJSR) \\ ISSN (Online): 2319-7064}

Index Copernicus Value (2013): 6.14 | Impact Factor (2014): 5.611

Ricci Scalar is given by

$$
R=\frac{f^{\prime \prime}}{f}+\frac{g^{\prime \prime}}{g}+\frac{l^{\prime \prime}}{l}-\frac{f^{\prime 2}}{2 f^{2}}-\frac{g^{\prime 2}}{2 g^{2}}-\frac{l^{\prime 2}}{4 l^{2}}+\frac{f^{\prime} l^{\prime}}{2 f l}+\frac{f^{\prime} g^{\prime}}{2 g f}+\frac{g^{\prime} l^{\prime}}{2 g l}
$$

Then using Einstein Field Equations (1), non-zero components of stress-energy tensor $T_{i j}$ are

$$
\begin{aligned}
& T_{00}=T_{0}=\frac{1}{\kappa}\left[\frac{g^{\prime \prime} f}{2 g}+\frac{l^{\prime \prime} f}{2 l}-\frac{g^{\prime 2} f}{4 g^{2}}-\frac{l^{\prime 2}}{8 l^{2}}+\frac{f^{\prime} g^{\prime} l^{\prime}}{g l}\right], \\
& T_{11}=T_{1}=-\frac{1}{\kappa}\left[\frac{l^{\prime 2}}{4 l^{2}}+\frac{f^{\prime} l^{\prime}}{4 f l}+\frac{g^{\prime} l^{\prime}}{4 g l}+\frac{f^{\prime} g^{\prime}}{4 f g}\right], \\
& T_{22}=T_{2}=\frac{1}{2 \kappa}\left[\frac{f^{\prime} g}{2 f^{2}}-\frac{f^{\prime \prime} g}{f}-\frac{l^{\prime \prime} g}{l}+\frac{l^{2} g}{4 l^{2}}-\frac{f^{\prime} g l^{\prime}}{2 f l}\right], \\
& T_{33}=T_{3}=\frac{1}{2 \kappa}\left[\frac{l^{\prime}}{4 l}-\frac{l f^{\prime \prime}}{f}-\frac{g^{\prime \prime} l}{g}+\frac{g^{\prime 2} l}{2 g^{2}}-\frac{f^{\prime} g^{\prime}}{2 g f l}\right] .
\end{aligned}
$$

Now, using equation (2), we have MC equations

$$
\begin{aligned}
& T_{0,1} X^{1}+2 T_{0} X^{0}{ }_{, 0}=0, \\
& T_{0} X^{0}{ }_{, 1}+T_{1} X^{1}{ }_{, 0}=0 \\
& T_{0} X^{0}{ }_{, 2}+T_{2} X^{2}{ }_{, 0}=0 \\
& T_{0} X^{0}{ }_{, 3}+T_{3} X^{3}{ }_{, 0}=0 \\
& T_{1,1} X^{1}+2 T_{1} X^{1}{ }_{, 1}=0 \\
& T_{1} X^{1}{ }_{, 2}+T_{2} X^{2}{ }_{, 1}=0 \\
& T_{1} X^{1}{ }_{, 3}+T_{3} X^{3}{ }_{, 1}=0 \\
& T_{2,1} X^{1}+2 T_{2} X^{2}{ }_{, 2}=0 \\
& T_{2} X^{2}{ }_{, 3}+T_{3} X^{3}{ }_{, 2}=0, \\
& T_{3,1} X^{1}+2 T_{3} X^{3}{ }_{, 3}=0,
\end{aligned}
$$

where comma denotes partial derivative and indices $0,1,2,3$ corresponds to the variables $t, \varrho, z, \phi$ respectively and $T_{i i}=$ $T_{i}(i=0,1,2,3)$.

\subsection{Degenerate Matter Symmetries}

In this section due to degeneracy of energy momentum tensor i.e. $\operatorname{det}\left(T_{a b}\right)=0$ we have the following different possibilities

a) for any $a, T_{a}=0$,

b) when only one $T_{a} \neq 0$,

c) when two $T_{a} \neq 0$,

d) when three $T_{a} \neq 0$.

Case (a):

This is trivial case. The MC equation (14)-(23) satisfied identically and thus every vector is matter collineations (symmetries) MC.

Case (b):

In this case, we have the following sub-cases,

i) $T_{0} \neq 0, T_{1}=T_{2}=T_{3}=0$,

ii) $T_{1} \neq 0, T_{0}=T_{2}=T_{3}=0$,

iii) $T_{2} \neq 0, T_{0}=T_{1}=T_{3}=0$,

iv) $T_{3} \neq 0, T_{0}=T_{1}=T_{2}=0$.

For sub-case b-(i), using equations (14)-(23), we have the following matter symmetries (collineations) i.e $\mathrm{MC}$ equations

$$
T_{0,1} X^{1}+2 T_{0} X_{, 0}^{0}=0
$$$$
T_{0} X^{0}{ }_{a}=0,(a=1,2,3)
$$

which has solution as $X^{0}=c_{1}, X^{1}=0$

For sub-case b-(ii), MC equations (14)-(23) becomes,

$$
\begin{aligned}
& T_{1} X^{1}{ }_{, b}=0,(b=0,2,3) \\
& T_{1,1} X^{1}+2 T_{1} X^{1}{ }_{, 1}=0 .
\end{aligned}
$$

Solving above equation, we get, $X^{1}=\frac{c_{1}}{\sqrt{T_{1}}}, X^{b}=X^{b}(t, \varrho, z, \phi)(b=0,2,3)$.
Now, constraint equations for sub-case b-(iii) using equations (14)-(23) are

$T_{2} X^{2}, a=0,(a=0,1,3)$

$T_{2,1} X^{1}+2 T_{2} X_{, 2}^{2}=0$.

Thus required solutions are

$X^{1}=-\frac{2 T_{2}}{T_{2,1}} F^{\prime}(z)$ and $X^{2}=F(z)$,

where $F(z)$ is an arbitrary function of $z$.

For sub-case b-(iv), we get similar type of solutions as in case b-(i) and these are

$X^{1}=0, X^{3}=c_{1}, X^{a}=X^{a}(t, \varrho, z, \phi)(a=0,2)$.

\section{Case (c):}

In this case, we have following possibilities
i) $T_{0} \neq 0, T_{1} \neq 0, T_{2}=0, T_{3}=0$,
ii) $T_{0} \neq 0, T_{1}=0, T_{2} \neq 0, T_{3}=0$,
iii) $T_{0} \neq 0, T_{1}=0, T_{2}=0, T_{3} \neq 0$,
iv) $T_{0}=0, T_{1} \neq 0, T_{2}=0, T_{3} \neq 0$,
v) $T_{0}=0, T_{1}=0, T_{2} \neq 0, T_{3} \neq 0$,
vi) $T_{0}=0, T_{1} \neq 0, T_{2} \neq 0, T_{3}=0$.

For sub-case c-(i), using equations (14)-(23), we have the following constraint equations

$$
\begin{aligned}
& T_{0,1} X^{1}+2 T_{0} X^{0}{ }_{, 0}=0, \\
& T_{0} X^{0}{ }_{, 1}+T_{1} X^{1}{ }_{, 0}=0, \\
& T_{0} X^{0}{ }_{, a}=0,(a=2,3) \\
& T_{1,1} X^{1}+2 T_{1} X^{1}{ }_{, 1}=0, \\
& T_{1} X^{1}{ }_{, b}=0,(b=2,3) .
\end{aligned}
$$

$X^{0}=F(t, \varrho), X^{1}=G(t, \varrho)$.

Equation (31), $X^{1}=\frac{c_{1}}{\sqrt{T_{1}}}$.

Using equation (28), (29) and (33), we have,

$X^{0}=\frac{T_{0,1}}{T_{0} \sqrt{T_{1}}} t c_{2}+c_{3}$,

where $c_{1}, c_{2}, c_{3}$ are constants of integration.

For sub-cases c-(ii), matter collineation equations (14)-(23) becomes

$$
\begin{aligned}
& T_{0,1} X^{1}+2 T_{0} X^{0}{ }_{, 0}=0, \\
& T_{0} X^{0}{ }_{, 2}+T_{2} X^{2}{ }_{, 0}=0, \\
& T_{2,1} X^{1}+2 T_{2} X^{2}, 2=0, \\
& T_{a} X^{a}{ }_{, b}=0,(a=0,2 \text { and } b=1,3) .
\end{aligned}
$$

On solving above equations, we arrive at solutions as $X^{0}=c_{1}, X^{1}=X^{1}(t, \varrho, z, \phi), X^{2}=c_{2}, X^{3}=X^{3}(t, \varrho, z, \phi)$.

Now, MC equations for sub-cases c-(iii) are given by

$$
\begin{aligned}
& T_{0,1} X^{1}+2 T_{0} X^{0}{ }_{, 0}=0, \\
& T_{0} X^{0}{ }_{, 3}+T_{3} X^{3}{ }_{, 0}=0, \\
& T_{3,1} X^{1}+2 T_{3} X^{3}{ }_{, 3}=0, \\
& T_{a} X^{a}{ }_{, b}=0(a=0,3 \text { and } b=1,2) .
\end{aligned}
$$

Using equations (38)-(41), we have solutions as $X^{0}=c_{1}, X^{1}=-\frac{2 T_{3}}{T_{3,1}} G^{\prime}(\phi), X^{2}=X^{2}(t, \varrho, z, \phi)$,

$X^{3}=G(\phi)$,

where $G(\phi)$ is an arbitrary function.

For sub-case c-(iv), using equations (14)-(23), constraint equations are

$$
\begin{aligned}
& T_{1,1} X^{1}+2 T_{1} X^{1}{ }_{, 1}=0 \\
& T_{1} X^{1}{ }_{, 3}+T_{3} X^{3}{ }_{, 1}=0, \\
& T_{3,1} X^{1}+2 T_{3} X^{3}{ }_{, 3}=0, \\
& T_{a} X^{a}{ }_{, b}=0,(a=1,3 \text { and } b=0,2) .
\end{aligned}
$$

Equation (42) and (45) gives, $X^{1}=F(\varrho, \phi), X^{3}=G(\varrho, \phi)$ and 


\section{International Journal of Science and Research (IJSR) \\ ISSN (Online): 2319-7064}

Index Copernicus Value (2013): 6.14 | Impact Factor (2014): 5.611

$X^{1}=\frac{c_{1}}{\sqrt{T_{1}}} .(46)$

Now, using (45) and (46), we get

$X^{3}=\frac{T_{3,1}}{2 T_{3} \sqrt{T_{1}}} \phi c_{2}+c_{3}, X^{0}=V^{0}(t, \varrho, z, \phi)$ and

$X^{2}=X^{2}(t, \varrho, z, \phi)$.

Now, for sub-case c-(v), using equations (14)-(23), we have

$$
\begin{aligned}
& T_{a} X^{a}{ }_{, b}=0(a=2,3 \text { and } b=0,1), \\
& T_{2,1} X^{1}+2 T_{2} X^{2}{ }_{, 2}=0, \\
& T_{2} X^{2}{ }_{, 3}+T_{3} X^{3}{ }_{, 2}=0, \\
& T_{3,1} X^{1}+2 T_{3} X^{3}{ }_{, 3}=0 .
\end{aligned}
$$

Therefore we have solutions as

$X^{1}=X^{1}(t, \varrho, z, \phi), \quad X^{2}=c_{1}, \quad X^{3}=c_{2} \quad$ with constraint condition $T_{2}=$ const. $\neq 0, T_{3}=$ const. $\neq 0$.

Now, for sub-case c-(vi), we have the following solutions as $X^{1}=\frac{c_{1}}{\sqrt{T_{1}}}, X^{2}=-\frac{T_{2,1}}{T_{2} \sqrt{T_{1}}} Z c_{1}+c_{2}$, $X^{0}=X^{0}(t, \varrho, z, \phi), X^{3}=X^{3}(t, \varrho, z, \phi)$.

Consider, following sub-cases for case (d)
i) $\quad T_{0} \neq 0, T_{1} \neq 0, T_{2} \neq 0, T_{3}=0$
ii) $\quad T_{0} \neq 0, T_{1} \neq 0, T_{2}=0, T_{3} \neq 0$,
iii) $\quad T_{0} \neq 0, T_{1}=0, T_{2} \neq 0, T_{3} \neq 0$,
iv) $\quad T_{0}=0, T_{1} \neq 0, T_{2} \neq 0, T_{3} \neq 0$

For sub-case d-(i), using equations (14)-(23), we have following $\mathrm{MC}$ equations

$$
\begin{aligned}
& T_{0,1} X^{1}+2 T_{0} X^{0}{ }_{, 0}=0, \\
& T_{0} X^{0}{ }_{, 1}+T_{1} X^{1}{ }^{, 0}=0, \\
& T_{0} X^{0}{ }_{, 2}+T_{2} X^{2}{ }_{, 0}=0, \\
& T_{1,1} X^{1}+2 T_{1} X^{1}{ }_{, 1}=0, \\
& T_{1} X^{1}{ }_{, 2}+T_{2} X^{2}{ }_{, 1}=0, \\
& T_{2,1} X^{1}+2 T_{2} X^{2}{ }_{, 2}=0, \\
& T_{a} X^{a}{ }_{, 3}=0,(a=0,1,2) .
\end{aligned}
$$

(56) $\quad X_{(4)}=-\frac{2 T_{3}}{T_{31}} z \frac{\partial}{\partial \rho}+\frac{T_{3} \phi^{2}}{T_{2}} \frac{\partial}{\partial z}+z \phi \frac{\partial}{\partial \phi}$,

We arrive at the solution, $X^{2}=c_{1}$.

Equation (54) implies, $X^{1}=\frac{G(t)}{\sqrt{T_{1}}}$ where $G(t)$ is an arbitary function. (58)

Now, using (52) and (58)

$$
X^{0}=-\dot{G}(t) \int \frac{\sqrt{T_{1}}}{T_{0}} d \varrho+c_{2} .
$$

Now, using (51) and (58) yields

$$
\frac{T_{0,1}}{2 T_{0} F(\varrho) \sqrt{T_{1}}}=\frac{\ddot{G}(t)}{G(t)}=\alpha^{2} \text { (const.), }
$$

where $F(\varrho)=\int \frac{\sqrt{T_{1}}}{T_{0}} d \varrho$.

When $\alpha^{2}=0, G(t)=c_{3} t+c_{4}$.

When $\alpha^{2}>0, G(t)=c_{3} e^{\alpha t}+c_{4} e^{-\alpha t}$

When $\alpha^{2}<0, G(t)=c_{3} \cos (\alpha t)+c_{4} \sin (\alpha t)$.

Apply same procedure as in case d-(i), we have following solutions for sub-case d-(ii), $X^{3}=c_{1}, X^{0}=$

$-\dot{G}(t) \int \frac{\sqrt{T_{1}}}{T_{0}} d \varrho+c_{2}$ and $X^{1}=\frac{G(t)}{\sqrt{T_{1}}}$.

where $G(t)=c_{3} t+c_{4}$ for $\alpha^{2}=0$,

$G(t)=c_{3} e^{\alpha t}+c_{4} e^{-\alpha t}$ for $\alpha^{2}>0$

$G(t)=c_{3} \cos (\alpha t)+c_{4} \sin (\alpha t)$ for $\alpha^{2}<0$.

Now, using equations (14)-(23), MC equations for sub-case d-(iii),

$$
\begin{aligned}
& T_{0,1} X^{1}+2 T_{0} X^{0}{ }_{, 0}=0, \\
& T_{0} X^{0}{ }_{, 2}+T_{2} X^{2}{ }_{, 0}=0, \\
& T_{0} X^{0}{ }_{, 3}+T_{3} X^{3}{ }_{, 0}=0, \\
& T_{2,1} X^{1}+2 T_{2} X^{2}{ }_{, 2}=0, \\
& T_{3,1} X^{1}+2 T_{3} X^{3}{ }_{, 3}=0, \\
& T_{2} X^{2}{ }_{, 3}+T_{3} X^{3}{ }_{, 2}=0, \\
& T_{a} X^{a}{ }_{, 1}=0(a=0,2,3)
\end{aligned}
$$

Therefore, $X^{0}=c_{0}, X^{2}=H(z, \phi), X^{3}=G(z, \phi)$.

Now, using equations (64), (65) and (66)

$$
-\frac{T_{2,1}}{T_{3,1} T_{3}} X^{3}, 33=X^{3}, 22 \text {. }
$$

Set $X^{3}=X(z) Y(\phi)$ then equation (68) gives

$$
-\frac{T_{2,1}}{T_{3,1} T_{3}} \frac{Y, 33}{Y}=\frac{X, 22}{X}=\alpha^{2} \text { (const.) }
$$

Therefore, for $\alpha^{2}=0$

$X^{1}=-\frac{2 T_{3}}{T_{3,1}}\left[c_{1} z+c_{3}\right]$,

$X^{2}=-\frac{T_{3}}{T_{2}} \phi\left[\frac{c_{1} \phi}{2}+c_{2}\right]+c_{5}$,

$X^{3}=c_{1} z \phi+c_{2} z+c_{3} \phi+c_{4}$.

for $\alpha^{2} \neq 0$

$X^{1}=-\frac{2 T_{3}}{T_{3,1}} \zeta \cdot \alpha\left\{\cos (\zeta \cdot \alpha) \phi\left[c_{2} e^{\alpha z}+c_{4} e^{-\alpha z}\right]-\right.$ $\left.\sin (\zeta . \alpha) \phi\left[c_{1} e^{\alpha z}+c_{3} e^{-\alpha z}\right]\right\}$,

$X^{2}=\frac{T_{3}}{T_{2}} \frac{\alpha}{\zeta}\left\{\sin (\zeta \alpha) \phi\left[c_{1} e^{\alpha z}-c_{3} e^{-\alpha z}\right]-\right.$

$\left.\cos (\zeta \alpha) \phi\left[c_{2} e^{\alpha z}-c_{4} e^{-\alpha z}\right]\right\}+c_{5}$,

$X^{3}=\cos (\zeta \alpha) \phi\left[c_{1} e^{\alpha z}+c_{3} e^{-\alpha z}\right]+\sin (\zeta \alpha) \phi\left[c_{2} e^{\alpha z}+\right.$ $\left.c_{4} e^{-\alpha z}\right]+c_{6}$.

Therefore matter symmetries for $\alpha^{2}=0$ are given by $X=$ $X^{i} \frac{\partial}{\partial x^{i}}, i=0,1,2,3$ i.e.

$X_{(1)}=\frac{\partial}{\partial t}, X_{(2)}=\frac{\partial}{\partial z}, X_{(3)}=\frac{\partial}{\partial \phi}$,

$X_{(5)}=\frac{T_{3} \phi}{T_{2}} \frac{\partial}{\partial z}+z \frac{\partial}{\partial \phi}, X_{(6)}=-\frac{2 T_{3}}{T_{3,1}} \frac{\partial}{\partial \varrho}+\phi \frac{\partial}{\partial \phi}$.

Then matter symmetries for $\alpha^{2} \neq 0$ are

$X_{(1)}=\frac{\partial}{\partial t}, X_{(2)}=\frac{\partial}{\partial z}, X_{(3)}=\frac{\partial}{\partial \phi}$,

$X_{(4)}=\phi e^{\alpha z}\left[\cos (\zeta \alpha) \frac{\partial}{\partial \phi}+\frac{T_{3}}{T_{2}} \frac{\alpha}{\zeta} \sin (\zeta \alpha) \frac{\partial}{\partial z}+\right.$ $\left.\frac{2 T_{3}}{T_{3,1}} \zeta \alpha \sin (\zeta \alpha) \frac{\partial}{\partial \varrho}\right]$

$X_{(5)}=\phi e^{\alpha z}\left[\sin (\zeta \alpha) \frac{\partial}{\partial \phi}+\frac{T_{3}}{T_{2}} \frac{\alpha}{\zeta} \cos (\zeta \alpha) \frac{\partial}{\partial z}-\right.$

$\left.\frac{2 T_{3}}{T_{3,1}} \zeta \alpha \cos (\zeta \alpha) \frac{\partial}{\partial \varrho}\right]$,

$X_{(6)}=\phi e^{-\alpha z}\left[\cos (\zeta \alpha) \frac{\partial}{\partial \phi}-\frac{T_{3}}{T_{2}} \frac{\alpha}{\zeta} \sin (\zeta \alpha) \frac{\partial}{\partial z}+\right.$

$\left.\frac{2 T_{3}}{T_{3,1}} \zeta \alpha \sin (\zeta \alpha) \frac{\partial}{\partial \varrho}\right]$,

$X_{(7)}=\phi e^{-\alpha z}\left[\sin (\zeta \alpha) \frac{\partial}{\partial \phi}+\frac{T_{3}}{T_{2}} \frac{\alpha}{\zeta} \cos (\zeta \alpha) \frac{\partial}{\partial z}-\right.$

$\left.\frac{2 T_{3}}{T_{3,1}} \zeta \alpha \sin (\zeta \alpha) \frac{\partial}{\partial \varrho}\right]$,

where $\sqrt{\frac{T_{3,1}}{T_{2,1}} T_{3}}=\zeta$.

For sub-case d-(iv), we have solutions as

$X^{0}=0, X^{2}=c_{1}, X^{3}=c_{2}, X^{0}=X^{0}(t, \varrho, z, \phi)$

\subsection{Non-Degenerate Matter Symmetries}




\section{International Journal of Science and Research (IJSR) \\ ISSN (Online): 2319-7064 \\ Index Copernicus Value (2013): 6.14 | Impact Factor (2014): 5.611}

In this case, $\operatorname{det}\left(T_{a b}\right) \neq 0$ which is possible when we take $T_{0}=\widetilde{c_{1}} \neq 0, T_{1}=\widetilde{c_{2}} \neq 0, T_{2}=\widetilde{c_{3}} \neq 0, T_{3}=\widetilde{c_{4}} \neq 0$ where $\widetilde{c_{1}}, \widetilde{c_{2}}, \widetilde{c_{3}}, \widetilde{c_{4}}$ are constants.

Therefore equations (14)-(23) become

$$
\begin{aligned}
& X^{a}{ }_{, a}=0(a=0,1,2,3), \\
& \widetilde{c_{1}} X^{0}{ }_{, 1}+\widetilde{c_{2}} X^{1}{ }_{, 0}=0, \\
& \widetilde{c_{1}} X^{0}{ }_{, 2}+\widetilde{c_{3}} X^{2}{ }_{, 0}=0, \\
& \widetilde{c_{1}} X^{0}{ }^{,}+\widetilde{c_{4}} X^{3}{ }_{, 0}=0, \\
& \widetilde{c_{2}} X^{1}{ }_{, 2}+\widetilde{c_{3}} X^{2}{ }_{, 1}=0, \\
& \widetilde{c_{2}} X^{1}{ }_{, 3}+\widetilde{c_{4}} X^{3}{ }_{, 1}=0, \\
& \widetilde{c_{3}} X^{2}{ }_{, 3}+\widetilde{c_{4}} X^{3}{ }_{, 2}=0 .
\end{aligned}
$$

Equation (70) implies $X^{0}=F(\varrho, z, \phi)$,

$X^{1}=G(t, z, \phi), X^{2}=H(t, \varrho, \phi), X^{3}=I(t, \varrho, z)$.

Now, using equations (74), (75) and (76), we get

$X^{3}{ }_{, 21}=0$ gives $X^{3}=c_{1} \varrho+c_{2}$. Substitute $X^{3}$ in equation (76) we get

$$
X^{2}=c_{3} t+c_{4} \text {. }
$$

Using equations (71)-(73) and (75) give

$X^{1}=c_{5} \phi$ and $X^{0}=c_{6} t z+c_{7}$

where $c_{1}, c_{2}, c_{3}, c_{4}, c_{5}=\frac{-\widetilde{c_{4}}}{\widetilde{c_{2}}} c_{1}, c_{6}=\frac{-\widetilde{c_{3}}}{\widetilde{c_{1}}}$ and $c_{7}$ are nonzero constants.

Then matter symmetries are given by

$X_{(1)}=\frac{\partial}{\partial t}, X_{(2)}=\frac{\partial}{\partial z}, X_{(3)}=\frac{\partial}{\partial \phi}, X_{(4)}=\varrho \frac{\partial}{\partial \phi}, X_{(5)}=t \frac{\partial}{\partial z}$, $X_{(6)}=\phi \frac{\partial}{\partial \varrho}, X_{(7)}=z \frac{\partial}{\partial t}$.

\section{Discussion}

In the classification of Linet-Tian space-time according to energy-momentum tensor, we find ten matter symmetries equations. We have solved these equations for degenerate case (section 2.1) where $\operatorname{det}\left(T_{a b}\right)=0$ as well as for nondegenerate case (section 2.2) when $\operatorname{det}\left(T_{a b}\right)=T_{0} T_{1} T_{2} T_{3} \neq$ 0 . From these equations we obtain different constraint equations on energy-momentum tensor. If we solve these constraint equations we can have new class of exact solutions of Einstein's field equations. It is observed from section (2.2) that when energy-momentum tensor is degenerate, then we obtain the four cases (a), (b), (c) and (d) where the matter symmetries equations admit infinite dimensional for all cases. It is very interesting to note that we have found a case (2.1.d.iii) where energy-momentum tensor is degenerate but the group of matter symmetries is finite dimensional i.e. there are six or seven independent MC's.

Furthermore, it is observed from section (2.2) that when energy-momentum tensor is non-degenerate we found MC's of seven dimensional. These include three usual Killing vectors for Linet-Tian space-time and the remaining are nontrivial MC's. All these results have been summarised in the table at the end of this conclusion. (see Appendix A)

\section{Conclusion}

In this paper, we have studied the symmetries of the energymomentum tensor $\left(T_{a b}\right)$ for the Linet-Tian metric. We have

classified Linet-Tian metric according to their matter symmetry by solving matter symmetries (collineation) equations. These matter symmetry equations are solved by taking one, two, three and four non-zero components of energy-momentum tensor $\left(T_{a b}\right)$ for the Linet-Tian spacetime which on solving gives the degenerate as well as nondegenerate cases. In degenerate case, we have obtained infinite number of MC's in all cases except the case-2.1-d(iii) which is degenerate but finite dimensional i.e. six or

\begin{tabular}{|c|c|c|}
\hline Case & Constraint & MC's \\
\hline 2.1.a & $T_{0}=T_{1}=T_{2}=T_{3}=0$ & Every vector \\
\hline 2.1.b.i & $T_{0} \neq 0, T_{1}=T_{2}=T_{3}=0$ & $\begin{array}{c}\text { Infinite } \\
\text { dimensional }\end{array}$ \\
\hline 2.1.b.ii & $T_{1} \neq 0, T_{0}=T_{2}=T_{3}=0$ & $\begin{array}{c}\text { Infinite } \\
\text { dimensional }\end{array}$ \\
\hline 2.1.b.iii & $T_{2} \neq 0, T_{0}=T_{1}=T_{3}=0$ & $\begin{array}{c}\text { Infinite } \\
\text { dimensional }\end{array}$ \\
\hline 2.1.b.iv & $T_{3} \neq 0, T_{0}=T_{1}=T_{2}=0$ & $\begin{array}{c}\text { Infinite } \\
\text { dimensional }\end{array}$ \\
\hline 2.1.c.i & $T_{0} \neq 0, T_{1} \neq 0, T_{2}=0, T_{3}=0$ & $\begin{array}{c}\text { Infinite } \\
\text { dimensional } \\
\end{array}$ \\
\hline 2.1.c.ii & $T_{0} \neq 0, T_{1}=0, T_{2} \neq 0, T_{3}=0$ & $\begin{array}{c}\text { Infinite } \\
\text { dimensional }\end{array}$ \\
\hline 2.1.c.iii & $T_{0} \neq 0, T_{1}=0, T_{2}=0, T_{3} \neq 0$ & $\begin{array}{c}\text { Infinite } \\
\text { dimensional } \\
\end{array}$ \\
\hline 2.1.c.iv & $T_{0}=0, T_{1} \neq 0, T_{2}=0, T_{3} \neq 0$ & $\begin{array}{c}\text { Infinite } \\
\text { dimensional } \\
\end{array}$ \\
\hline 2.1.c. $\mathrm{v}$ & $\begin{array}{c}T_{0}=0, T_{1}=0, T_{2}=\text { const. } \neq 0, \\
T_{3}=\text { const } . \neq 0\end{array}$ & $\begin{array}{c}\text { Infinite } \\
\text { dimensional } \\
\end{array}$ \\
\hline 2.1.c.vi & $T_{0}=0, T_{1} \neq 0, T_{2} \neq 0, T_{3}=0$ & $\begin{array}{c}\text { Infinite } \\
\text { dimensional } \\
\end{array}$ \\
\hline 2.1.d.i & $T_{0} \neq 0, T_{1} \neq 0, T_{2} \neq 0, T_{3}=0$ & $\begin{array}{c}\text { Infinite } \\
\text { dimensional } \\
\end{array}$ \\
\hline 2.1.d.ii & $T_{0} \neq 0, T_{1} \neq 0, T_{2}=0, T_{3} \neq 0$ & $\begin{array}{c}\text { Infinite } \\
\text { dimensional }\end{array}$ \\
\hline \multirow{2}{*}{ 2.1.d.iii } & $\begin{array}{c}T_{0} \neq 0, T_{1}=0, T_{2} \neq 0, T_{3} \neq 0 \\
\alpha^{2}=0\end{array}$ & 6 \\
\hline & $\begin{array}{c}T_{0} \neq 0, T_{1}=0, T_{2} \neq 0, T_{3} \neq 0 \\
\alpha^{2} \neq 0\end{array}$ & 7 \\
\hline 2.1.d.iv & $T_{0}=0, T_{1} \neq 0, T_{2} \neq 0, T_{3} \neq 0$ & $\begin{array}{c}\text { Infinite } \\
\text { dimensional } \\
\end{array}$ \\
\hline
\end{tabular}
seven and in non-degenerate case we have obtained seven finite dimensional MC's.

\section{Appendix A}

The usual linearly independent Killing vectors for LinetTian metric are given by

$$
\begin{aligned}
& X_{(1)}=\frac{\partial}{\partial t}, \\
& X_{(2)}=\frac{\partial}{\partial z}, \\
& X_{(3)}=\frac{\partial}{\partial \phi} .
\end{aligned}
$$

For Degenerate Case

\section{For Non-degenerate Case}

\begin{tabular}{|c|c|c|}
\hline Case & Constraint & MC's \\
\hline \multirow{2}{*}{2.2} & $\left.\begin{array}{l}T_{0}=\widetilde{c_{1}} \neq 0, T_{1}=\widetilde{c_{2}} \neq 0, \\
\\
T_{2}=\widetilde{c_{3}} \neq 0, T_{3}=\widetilde{c_{4}} \neq 0\end{array}\right] 7$ \\
\hline
\end{tabular}

\section{References}

\section{Volume 4 Issue 11, November 2015}




\section{International Journal of Science and Research (IJSR) \\ ISSN (Online): 2319-7064}

Index Copernicus Value (2013): 6.14 | Impact Factor (2014): 5.611

[1] S. Weinberg, "Gravitation and Cosmology - Principles and Applicatios of the General Theory of Relativity", John Wiley and Sons Inc., New York, 1972

[2] G.S. Hall, J. Math. Phys., 31, 1198 (1990)

[3] A. Melfo, L. Nunez, U. Percoco and V.M. Villalba, J. Math. Phys., 33, 2258 (1992).

[4] A.H. Bokhari and A. Qadir, J. Math. Phys., 34, 3543 (1993).

[5] H. Stephani, D. Kramer, M. MacCallum, C. Hoenselaers and E. Herlt, "Exact Solutions of Einstein's field equations", Cambridge University Press, 2nd edn., 2003

[6] G. S. Hall, I. Roy, and L. R. Vaz, Gen. Relativ. Gravit., 28, 299 (1996)

[7] U. Camci and A. Barnes, Class. Quantum Grav., 19, 393 (2002)

[8] J. Carot and J. da Costa, Proceedings of the 6th Canadian Conference on General Relativity and Relativistic Astrophysics, Fields Inst. Commun. 15 (American Mathematical Society WC, Providence, RI, 1997), pp. 179; J. Carot, J. da Costa, and E. G. L. R. Vaz, J. Math. Phys., 35, 4832 (1994).

[9] M. Tsamparlis and P. S. Apostolopoulos, J. Math. Phys., 41, 7573 (2000)

[10] M. Sharif, Nuovo Cimento Soc. Ital. Fis., B 116, 673 (2001); Astrophys. Space Sci., 278, 447 (2001)

[11] U. Camci and M. Sharif, Gen. Relativ. Gravit., 35, 97 (2003); Class. Quantum Grav., 20, 2169 (2003)

[12] M. Sharif and S. Aziz, Gen. Relativ. Gravit., 35, 1091 (2003);

[13] M. Sharif, J. Math. Phys., 44, 5141 (2003)

[14] M. Sharif, J. Math. Phys., 45, 1532 (2004)

[15] M. Sharif, arXiv:gr-qc/0702060v1 10 Feb 2007

[16] M.Salti, M. Korunur, I. Acikgoz, F. Binbay, N. Pirinccioglu, Rom. Journ. Phys., 59, 65 (2014)

[17] B. Linet, J. Math. Phys., 27, 1817 (1986)

[18] Q. Tian, Phys. Rev. D 33, 3549 (1986)

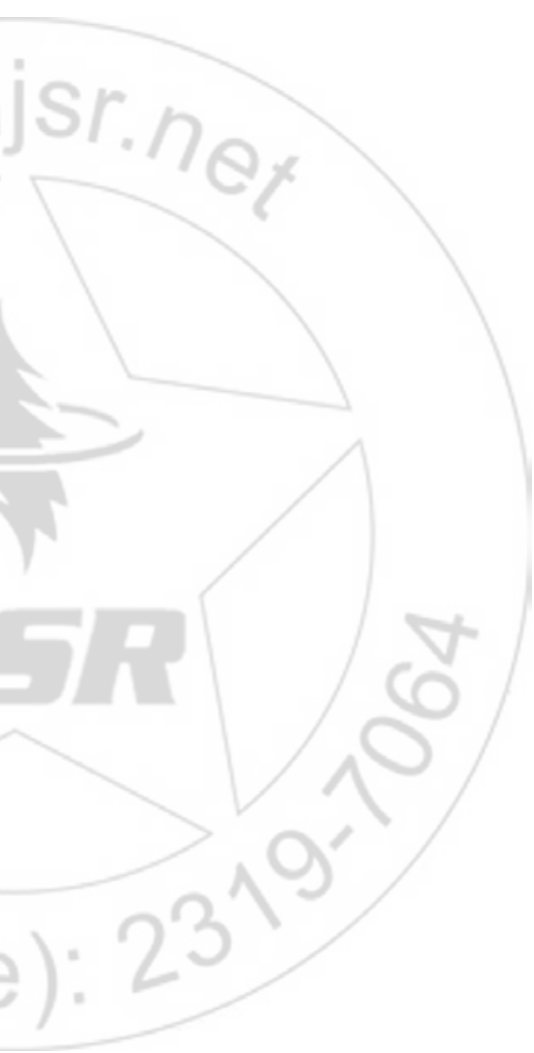

